\title{
A SYMMETRIC STAR POLYHEDRA THAT TILES BUT NOT AS A FUNDAMENTAL DOMAIN
}

\begin{abstract}
SÁNDOR SZABÓ
ABSTRACT. In [7] S. K. Stein constructed a 10-dimensional centrally-symmetric star body whose translates tile 10 -space but whose translates by a lattice do not tile it. In [8] he constructed a 5-dimensional star polyhedron whose translates tile 5-space but whose congruent copies by a group of motions do not tile it. So there is no lattice tiling by translates of this polyhedron. In the present paper we shall construct a 5-dimensional centrally-symmetric star polyhedron whose translates tile 5-space but whose congruent copies by a group of motions do not tile it. Furthermore, this phenomenon occurs at an infinitude of dimensions.
\end{abstract}

1. Preliminaries. Let $\mathscr{K}$ be a set in $n$-dimensional Euclidean space $R^{n}$ which is homeomorphic to an $n$-dimensional cube. If there exists a point $P$ in $\mathscr{K}$ such that for every point $Q$ in $\mathcal{K}$ the section $P Q$ lies in $\mathcal{K}$, then $\mathcal{K}$ is a star body. A system of congruent copies of $\mathscr{K}$, whose union is $R^{n}$ and whose interiors are disjoint, is a tiling.

The second part of the 18th problem of D. Hilbert is the following question [2]: "Whether polyhedra also exist which do not appear as fundamental regions by groups of motions, by means of which nevertheless by a suitable juxtaposition of congruent copies a complete filling up of all space possible?". In [2] K. Reinhard constructed a 3-dimensional polyhedron which showed that the answer is "yes". $\mathbf{J}$. Milnor gives reference for example in $R^{2}$ star, not symmetric. In [8] S. K. Stein constructed a 5-dimensional star polyhedron with this property. Furthermore, all motions in his example are translations. We shall construct a 5-dimensional centrally-symmetric star polyhedron with this property.

Another problem in the field of the geometry of numbers is the following question. If translates of a centrally-symmetric star body in Euclidean space can be packed with a certain density, is it possible to find a lattice packing by translates of it that is at least as dense? In [7] S. K. Stein constructed a 10-dimensional body which showed that the answer is "no". We shall construct a 5-dimensional polyhedron with this property.

2. The Theorem. We shall use the following lemmas. Reference [9] contains proofs of these lemmas.

LEMMA 1 (S. K. ZAREMBA [11]). If $q, r, s$ are integers such that $q$ is a power of $a$ prime and $r \geqslant 1$ and $s=\left(q^{r}-1\right) /(q-1)$, then for an $s$-dimensional chess board of

Received by the editors September 15, 1982 and, in revised form, January 20, 1983.

1980 Mathematics Subject Classification. Primary 05B45; Secondary 20K01, 52A45.

Key words and phrases. Tiling, star body, factorization of finite abelian groups, groups of motions, lattice. 
side $q$ there exists a set of castles that attack each unoccupied position of the chess board exactly once.

LEMMA 2 (R. BAER [1]). If a finite abelian group is expressible as the union of at least two subgroups, any two of which meet only at $\{0\}$, then every nonzero element of the group has the same order.

THEOREM. If $q, r, s$ are integers such that $q$ is a power of a prime and it is not a prime and $r \geqslant 2$ and $s=\left(q^{r}-1\right) /(q-1)$, then there exists an $s$-dimensional centrally-symmetric star polyhedron whose translates tile s-space but whose congruent copies by a group of motions do not tile it.

Proof. We shall generalize S. K. Stein's method. Denote by $R^{n}, n$-dimensional Euclidean space. Let $O$ be a fixed point in $R^{n}$ and let $e_{1}, \ldots, e_{n}$ be a fixed orthonormal basis in $R^{n}$. Let $\lambda$ be a positive real number and $k$ an integer such that $1 \leqslant k \leqslant n$. Consider the $n$-dimensional cell

$$
\mathbb{Q}_{k}^{\lambda}:=\left\{P: \overrightarrow{O P}=a_{1} e_{1}+\cdots+a_{n} e_{n},\left|a_{k}\right| \leqslant \lambda+\frac{1}{2},\left|a_{i}\right| \leqslant \frac{1}{2}, i \in\{1, \ldots, n\} \backslash\{k\}\right\} .
$$

The point $O$ is its center and its edges are parallel to the coordinate unit vectors $e_{1}, \ldots, e_{n}$ and their lengths are $1, \ldots, 1,2 \lambda+1,1, \ldots, 1$, respectively. $2 \lambda+1$ is in the $k$ th place.

The set $\cup_{i=1}^{n} \mathbb{Q}_{i}^{\lambda}$ is called a $(\lambda, n)$ cross which consists of the central cube $\cap_{i=1}^{n} \mathbb{Q}_{i}^{\lambda}=\mathbb{Q}_{i}^{\lambda} \cap \mathbb{Q}_{j}^{\lambda}, i \neq j$, and $2 n$ arms of length $\lambda$. The union of the translates of the sets $\mathbb{Q}_{1}^{\lambda}, \ldots, \mathbb{Q}_{n}^{\lambda}$ by the vectors $\lambda e_{1}, \ldots, \lambda e_{n}$, respectively, is called a $(2 \lambda, n)$ semicross which consists of a central cube and $n$ arms of length $2 \lambda$.

Let $q, r, s$ be integers under the same hypotheses as in Lemma 1 and let $L^{\prime \prime}$ be a lattice spanned by $q e_{1}, \ldots, q e_{s}$. The translates of a cube of edge $q$ (which is identified with the chess board) by the vectors of $L^{\prime \prime}$ tile $R^{s}$. The chess board consists of $q^{s}$ cubes of edge 1 . So the translates of a unit cube of the chess board tile $R^{s}$. Their translations form a lattice $L$ which is spanned by $e_{1}, \ldots, e_{s}$. Consider the union of the translates by the vectors of $L^{\prime \prime}$ the $s q+1$ cubes which are attacked by a castle. Since its translates tile $R^{s}$ and it can be divided into $\left(\frac{1}{2}(q-1), s\right)$ crosses and similarly, it can be divided into $(q-1, s)$ semicrosses so there exists a tiling by translates of a $\left(\frac{1}{2}(q-1), s\right)$ cross and there exists a tiling by translates of a $(q-1, s)$ semicross.

Now we shall modify the $\left(\frac{1}{2}(q-1), s\right)$ cross. It can be done in such a way that

(i) the modified $\left(\frac{1}{2}(q-1), s\right)$ cross is a star body and it is a centrally-symmetric polyhedron,

(ii) translates of a modified cross tile $R^{s}$,

(iii) if congruent copies of a modified cross tile $R^{s}$, then they are its translates,

(iv) translations by the vectors $q e_{1}, \ldots, q e_{s}$ are elements of the motion group of every tiling by congruent copies of a modified $\left(\frac{1}{2}(q-1), s\right)$ cross.

Finally we shall prove that the congruent copies of a modified cross by a group of motions do not tile $R^{s}$. Consider a tiling by congruent copies of a modified $\left(\frac{1}{2}(q-1), s\right)$ cross. In virtue of (iii) this tiling consists of translates of a modified 
$\left(\frac{1}{2}(q-1), s\right)$ cross. Assume that the translations form a group and denote this group by $L^{\prime}$. In virtue of (iv) $q e_{1}, \ldots, q e_{s}$ are elements of $L^{\prime}$. Then there exists a lattice tiling by translates of a $\left(\frac{1}{2}(q-1), s\right)$ cross and there exists a lattice tiling by translates of a $(q-1, s)$ semicross. This means that each $l$ in $L$ is uniquely expressible in the form $l=l^{\prime}+x$, where $l^{\prime} \in L^{\prime}$ and $x \in X:=\cup_{i=1}^{s}\left\{0, e_{i}, 2 e_{i}, \ldots,(q-1) e_{i}\right\}$. In other words, the equation $L=L^{\prime}+X$ is a factorization of $L$. Obviously, $L$ and $L^{\prime}$ are abelian groups under addition of vectors and $L^{\prime}$ is a subgroup of $L$. Denote by $G$ the factor group $L / L^{\prime}$ and denote by $g_{i}$ the coset of $L / L^{\prime}$ containing the element $e_{i}$. We have

$$
G=\bigcup_{i=1}^{s}\left\{0, g_{i}, 2 g_{i}, \ldots,(q-1) g_{i}\right\}
$$

and for $i \neq j$,

$$
\left\{0, g_{i}, 2 g_{i}, \ldots,(q-1) g_{i}\right\} \cap\left\{0, g_{j}, 2 g_{j}, \ldots,(q-1) g_{j}\right\}=\{0\}
$$

Since $q e_{i} \in L^{\prime}$ and $q^{\prime} e_{i} \notin L^{\prime}$ for $0<q^{\prime}<q$, the set $\left\{0, g_{i}, 2 g_{i}, \ldots,(q-1) g_{i}\right\}$ is a cyclic group of order $q$. If $q$ is not a prime, then $G$ has a nonzero element whose order is not $q$; if $r \geqslant 2$, then $s>2$. These violate Lemma 2 .

3. The alteration. Let $\bigodot$ be an $(s-1)$-dimensional cube in $R^{s}$ whose edges are parallel to the vectors $e_{1}, \ldots, e_{k-1}, e_{k+1}, \ldots, e_{s}$ and $s \geqslant 3$. Divide this cube into $2^{s-1}$ cubes $\bigodot_{1}, \ldots, e_{2^{s-1}}$. Denote their centers by $O_{1}, \ldots, O_{2^{s-1}}$. Assume that $\bigodot_{i} \cup \bigodot_{i+1}$ is centrally symmetric with respect to the center of $\mathcal{C}$. Translate the point $O_{i}$ by the vector $\mu_{i} e_{k}$ and translate the point $O_{i+1}$ by the vector $-\mu_{i} e_{k}$, where $\mu_{i}>0$, $i=1,3,5, \ldots, 2^{s-1}-1$. So we have points $O_{1}^{\prime}, \ldots, O_{2^{s-1}}^{\prime}$. Consider the convex hull of $\bigodot_{i}$ and $O_{i}^{\prime}$ for $i=1, \ldots, 2^{s-1}$. We have $2^{s-1} s$-dimensional pyramids. The union of the $(s-1)$-dimensional faces of these pyramids without the cubes $\bigodot_{1}, \ldots, \mathcal{C}_{2^{s-1}}$ is called the modified $C$ by the numbers $\mu_{i}$.

Let $\mu_{i j}, \nu_{i j}, \varepsilon$ be different real numbers such that $0<\mu_{i j}, \nu_{i j}<\varepsilon, i=1, \ldots, s$, $j=1,3,5, \ldots, 2^{s-1}-1$. The intersection of the $(s-1)$-dimensional plane, spanned by $e_{1}, \ldots, e_{k-1}, e_{k+1}, \ldots, e_{s}$ and the central cube of the $\left(\frac{1}{2}(q-1), s\right)$ cross, is an $(s-1)$-dimensional cube. Modify this cube by the numbers $\mu_{k j}$ and translate this modified face by the vectors $\pm \frac{1}{2} e_{k}, \ldots, \pm \frac{1}{2} q e_{k}$. Repeat this process for $k=1, \ldots, s$.

Divide the $\left(\frac{1}{2}(q-1), s\right)$ cross into $[s(q-1)+1] 2^{s}$ cubes of edge $\frac{1}{2}$. In this way we modified all faces of the cubes of the central cube of the cross and we modified two faces of the remaining cubes of the cross. Modify all of the faces of the cubes of the cross which is not modified and whose edges are parallel to the vectors $e_{1}, \ldots, e_{k-1}, e_{k+1}, \ldots, e_{s}$ by the numbers $\nu_{k j}$ in the same way. Repeat this process for $k=1, \ldots, s$.

Obviously, the number $\varepsilon$ can be selected such that the modified $\left(\frac{1}{2}(q-1), s\right)$ cross is a star body and it is a centrally-symmetric polyhedron.

For suitable $q, s$ there exists a tiling by translates of a $\left(\frac{1}{2}(q-1), s\right)$ cross in which an end of the arm of a cross always meets along an entire end of the arm of another cross. Modify all of the crosses of this system in the same way; we have a tiling by translates of a modified $\left(\frac{1}{2}(q-1), s\right)$ cross. 
Consider a tiling by congruent copies of a modified $\left(\frac{1}{2}(q-1), s\right)$ cross. Then the modified cubes of edge $\frac{1}{2}$ tile $R^{s}$. So the original cubes of edge $\frac{1}{2}$ tile $R^{s}$. In virtue of the modifying of the faces of the cubes, the neighbouring cubes meet along an entire $(s-1)$-dimensional face, that is, the modified $\left(\frac{1}{2}(q-1), s\right)$ crosses of the tiling are translates of a modified $\left(\frac{1}{2}(q-1), s\right)$ cross.

In virtue of the modifying of the faces of the central cube of the cross, an end of the arm of a modified cross always meets along an entire end of the arm of another modified cross. Thus we have (iv).

The author would like to thank the referee for his valuable suggestion.

\section{REFERENCES}

1. R. Baer, Partitionen endlichen Gruppen, Math. Z. 75 (1961), 333-372.

2. D. Hilbert, Mathematical problems, Lecture delivered before the International Congress of Mathematicians at Paris in 1900, Bull. Amer. Math. Soc. 8 (1901-1902), 437-479.

3. J. Milnor, Hilbert's problem 18: On crystalographic groups, fundamental domains, and on sphere packing, Proc. Sympos. Pure Math., vol. 28, Amer. Math. Soc., Providence, R. I., pp. 491-506.

4. K. Reinhard, Zur zerlegung der Euklidschen Räume in Kongruente Polytope, Sitzberichte Preuss. Akad. Wiss. (1928), 150-155.

5. C. A. Rogers, Packing and covering, Cambridge Tracts in Math. Phys., no. 54, Cambridge Univ. Press, New York, 1964.

6. S. K. Stein, Factoring by subsets, Pacific J. Math. 22 (1967), 523-541.

7. _ A symmetric star body that tiles but not as a lattice, Proc. Amer. Math. Soc. 36 (1972), $543-548$.

8. __ Tiling space by congruent polyhedra, Bull. Amer. Math. Soc. 80 (1974), 819-820.

9. Algebraic tiling, Amer. Math. Monthly 81 (1974), 445-462.

10. M. R. Von Wolf, A star domain with densest admissible point set not a lattice, Acta Math. 108 (1962), 53-60.

11. S. K. Zaremba, Covering problems concerning abelian groups, J. London Math. Soc. 27 (1952), 242-246.

12. H. Zassenhaus, Modern developments in the geometry of numbers, Bull. Amer. Math. Soc. 67 (1961), 427-439.

Department of Mathematics, Faculty of Civil Engineering, Technical University Budapest, H-1111 Budapest, StoczeK U. 2, Hungary 